\title{
Study of mode of delivery and maternal morbidity in preterm and term COVID-19 positive pregnant women
}

\author{
Saksha Dholakiya, Pooja S. Singh*, Jaishree Bamniya, Haresh U. Doshi
}

Department of Obstetrics and Gynaecology, GCS Medical College, Hospital and RC, Ahmedabad, Gujarat, India

Received: 21 November 2021

Accepted: 07 December 2021

\author{
*Correspondence: \\ Dr. Pooja S. Singh, \\ E-mail: drpsomesh03@yahoo.co.in
}

Copyright: (c) the author(s), publisher and licensee Medip Academy. This is an open-access article distributed under the terms of the Creative Commons Attribution Non-Commercial License, which permits unrestricted non-commercial use, distribution, and reproduction in any medium, provided the original work is properly cited.

\begin{abstract}
Background: Pregnant women are likely to represent a high-risk population during current coronavirus 2019 pandemic caused by severe acute respiratory syndrome coronavirus-2. The aim and objective of this study was to evaluate how COVID-19 pandemic has affected the mode of delivery and whether complications of the disease and mortality rate are higher in pregnant women than in non-pregnant women.

Methods: This ambispective observational study was conducted in department of obstetrics and gynaecology of our institute. Pregnant women diagnosed with positive for COVID-19 via the SARS-CoV-2 RT-PCR test in the third trimester and all neonates with complete COVID-19 testing and delivery data. This data was analysed.

Results: Out of total 66 cases studied, 48 patients $(72.7 \%)$ were asymptomatic, while $13(19.7 \%)$ had mild respiratory or gastro intestinal symptoms on initial assessment at admission, including cough, sore throat, fever, weakness or diarrhoea. Cesarean sections were performed in $57.6 \%$ of cases. There were no cases of maternal or neonatal mortality. Conclusions: The study revealed that COVID-19 positive pregnant women are usually asymptomatic or mildmoderately symptomatic, similar to COVID-19 positive non-pregnant women. There was a noted rise in the rate of caesarean sections as a mode of delivery.
\end{abstract}

Keywords: RT-PCR, COVID-19 and pregnancy, SARS-CoV-2

\section{INTRODUCTION}

The current outbreak of Coronavirus disease 2019 (COVID-19), caused by the Severe acute respiratory syndrome coronavirus 2 (SARS-CoV-2), was declared a pandemic by the WHO on March 11, 2020. ${ }^{1}$ The vulnerable population needs to be taken utmost care of, during this viral pandemic. Pregnant women are known to be affected more as compared to non-pregnant women by respiratory illnesses, resulting in increased infectious morbidity and high maternal mortality rates. With COVID-19 indiscriminate and sustained spread across the globe, we are likely to see women with COVID-19 canvassed across all trimesters of pregnancy. The second wave of COVID-19 proved more fatal as compared to first wave. Pregnant females are as vulnerable as compared to general population. $^{2}$
The most common outcome in pregnant women with COVID-19 was pneumonia and related complications. ${ }^{3}$

Pregnancy causes physiological changes in the respiratory and circulatory systems as well as alterations in immunological reactions increase the susceptibility of a pregnant woman to respiratory infections. ${ }^{4}$ The changes include reduced functional residual volumes, elevation of the diaphragm, relaxation of ligaments in the ribs, increased pulmonary hypertension resulting in hyperventilation or even hypoxic respiratory failure, and an immunocompromised state. Moreover, viral infection in pregnancy can result in modification of the cardiovascular system, increased metabolic rate and consumption of oxygen, higher pulmonary vascular resistance, and even heart failure. During the third trimester of pregnancy, due to increased maternal oxygen 
demands, physiological anemia, and supply of fetal oxygen, probability of physical dyspnea is high leading to further worsening of breathing difficulties. However, the existing data available so far do not clearly reflect an increased risk of COVID-19 complications in pregnant women, the unique immunologic changes of pregnancy are thought to suppress the virulence of the virus.

In this study we want to evaluate whether COVID-19 pandemic has affected the mode of delivery and whether complications of the disease and mortality rate are higher in pregnant women than in non-pregnant women. Also, whether there is a high chance of premature delivery or fetal mortality or vertical transmission of the disease needs to be answered. More research is required for planning effective obstetrical management for pregnant women with COVID-19. The concerns have been raised worldwide about the risk of intrauterine transmission of this virus from the mother to the fetus. Further studies are required to ascertain the possibility of transmission of COVID-19 in pregnant women vertically or during delivery.

\section{Aim and objectives}

The aim and objective of this study was to the mode of delivery in COVID-19 positive pregnant women and maternal morbidity and mortality and to investigate the outcomes in the delivered neonates, and incidence of vertical transmission of the corona virus disease 2019 based on early RNA detection of severe acute respiratory syndrome corona virus after birth from pharyngeal swab in neonates of COVID-19 positive pregnant women.

\section{METHODS}

This ambispective observational study was conducted in department of obstetrics and gynaecology of our institute from $1^{\text {st }}$ May 2020 to $30^{\text {th }}$ April 2021 after ethics committee approval and enrolment in ICMR research portal. The inclusion criteria were pregnant women diagnosed with positive for COVID-19 via the SARSCoV-2 RT-PCR test in the third trimester and all neonates with complete COVID-19-19 testing and delivery data. Those with maternal or neonatal records without SARSCoV-2 RT-PCR test results and neonates not delivered at our hospital were excluded. A total of 66 cases were studied. The suspected cases of COVID-19 infection were screened based on routine blood test in combination with symptoms such as fever, cough, chest tightness or gastrointestinal symptoms, and admitted to an isolated ward in our hospital. Two swabs were taken from upper and lower respirstory tract (naso-pharynx and oropharynx) from suspected cases and sent for RT-PCR on day of admission. ${ }^{5}$ Maternal demographic data collected included maternal age, gravity, parity, gestational age at delivery. And medical comorbidities such as hypertensive disorders, diabetes, asthma, Body mass index (BMI)>30 $\mathrm{kg} / \mathrm{m}^{2}$. The clinical characteristics of women diagnosed with COVID-19 included signs and symptoms such as cough, shortness of breath, gastrointestinal symptoms, fever (temperature of $38^{\circ} \mathrm{C}$ or greater) and laboratory criteria included maternal serum laboratory tests including white blood cell count, platelet count, CRP, D-dimer. Neonatal outcomes included maturity, birth weight, Apgar score, Neonatal intensive care unit (NICU) admission, and NICU length of stay. Neonatal complications included Transient tachypnoea of newborn (TTN), Respiratory distress syndrome (RDS), invasive mechanical ventilation, sepsis, and neonatal mortality. Other data collected included maternal and neonatal treatment progress, recovery and discharge information, treatment mode information, and death information.

\section{RESULTS}

In our study, a total of sixty-six pregnant women with COVID-19 were included. Gestations at diagnosis ranged from 28 weeks to 41 weeks. 11 patients (16.7\%) had comorbidities. All our patients were diagnosed with COVID-19 via RT-PCR, which is reverse transcription polymerase chain reaction test for qualitative detection of nucleic acids from SARS-CoV-2 in upper respiratory specimens and is currently the gold standard for testing COVID-19. 48 patients (72.7\%) were asymptomatic, while $13(19.7 \%)$ had mild respiratory or gastro intestinal symptoms on initial assessment at admission, including cough, sore throat, fever, weakness or diarrhoea. Symptomatic treatment was prescribed for upper respiratory tract symptoms and fever. Some women who were clinically stable with mild or no symptoms, but were still RT-PCR positive, were isolated and remained there until they were negative on RT-PCR test following national policy. Four patients had moderate severity of COVID-19 infection and 1 patient with severe COVID-19 required ICU admission. There were no cases of maternal mortality.

Table 1: Demographic data.

\begin{tabular}{|c|c|c|c|}
\hline Variables & & No. of cases & Percentage of total cases $(\mathrm{N}=66)(\%)$ \\
\hline \multirow{3}{*}{ Age (in years) } & 25 or less & 32 & 48.5 \\
\hline & $26-30$ & 21 & 31.8 \\
\hline & More than 30 & 13 & 19.7 \\
\hline \multirow{4}{*}{$\begin{array}{l}\text { Associated } \\
\text { comorbidity }\end{array}$} & Hypertensive disorders of pregnancy & 5 & 7.6 \\
\hline & Controlled hypothyroidism & 4 & 6.1 \\
\hline & GDM & 1 & 1.5 \\
\hline & SLE & 1 & 1.5 \\
\hline
\end{tabular}




\begin{tabular}{|llll|}
\hline Variables & & No. of cases & Percentage of total cases $(\mathbf{N}=\mathbf{6 6})(\boldsymbol{\%})$ \\
\hline \multirow{3}{*}{ Gravidity } & NONE & 55 & 83.3 \\
\hline & Primi & 17 & 25.7 \\
\cline { 2 - 4 } & Second & 24 & 36.4 \\
\hline
\end{tabular}

Table 2: COVID-19 infection in pregnant women.

Severity of COVID-19 infection

Asymptomatic COVID-19 positive

Mild (sore throat, fever, weakness, diarrhea)

Moderate (breathlessness)

Severe (pneumonia)

\begin{tabular}{ll} 
No. of cases & Percentage of total cases $(\mathrm{N}=66)(\%)$ \\
\hline 48 & 72.7 \\
13 & 19.7 \\
\hline 4 & 6.1 \\
\hline 1 & 1.5 \\
\hline
\end{tabular}

Table 3: Mode of delivery and vertical transmission.

\begin{tabular}{|lll|}
\hline Variables & No. of cases & Percentage of total cases $(\mathbf{N}=\mathbf{6 6})(\mathbf{\%})$ \\
\hline Vaginal delivery & 28 & 42.4 \\
\hline Caesarean delivery & 38 & 57.6 \\
\hline Term births & 54 & 81.8 \\
\hline Preterm births (weeks) & 12 & 18.2 \\
\hline$<28$ & 0 & 0 \\
\hline$<34$ & 5 & 7.6 \\
\hline$<37$ & 7 & 10.6 \\
\hline $\begin{array}{l}\text { Vertical transmission (SARS-CoV rTPCR positive } \\
\text { neonates) }\end{array}$ & 4 & 6.1 \\
\hline
\end{tabular}

Table 4: Indications of caesarean delivery.

\begin{tabular}{|lll|}
\hline Indications & No. of cases & $\begin{array}{l}\text { Percentage of total cesarean } \\
\text { deliveries }(\mathbf{N}=\mathbf{3 8})\end{array}$ \\
\hline Fetal distress & 9 & 13.6 \\
\hline Previous cesarean delivery & 9 & 13.6 \\
\hline Gestational hypertension/pre-eclampsia & 3 & 4.5 \\
\hline Oligohydramnios & 5 & 7.6 \\
\hline Twin gestation & 2 & 3.0 \\
\hline Breech presentation & 3 & 4.5 \\
\hline Precious pregnancy & 2 & 3.0 \\
\hline Abruptio placenta & 1 & 1.5 \\
\hline Demand cesarean in COVID-19 positive & 4 & 6.1 \\
\hline Total & 38 & 100 \\
\hline
\end{tabular}

Table 5: Neonatal outcomes in COVID-19 positive mothers.

\begin{tabular}{|c|c|c|c|}
\hline \multicolumn{2}{|l|}{ Variables } & \multirow{2}{*}{$\begin{array}{l}\text { No. of } \\
\text { neonates } \\
2\end{array}$} & \multirow{2}{*}{$\begin{array}{l}\text { Percentage of total cases }(\mathrm{N}=66) \\
3.0\end{array}$} \\
\hline \multirow{3}{*}{ Birth weight (kg) } & 1.5 or less & & \\
\hline & 2.5 or less & 15 & 22.7 \\
\hline & More than 2.5 & 49 & 74.2 \\
\hline $\begin{array}{l}\text { Apgar at } 5 \\
\text { minutes }\end{array}$ & Less than 7 & 1 & 1.5 \\
\hline \multirow{5}{*}{ NICU admission } & TTN & 3 & 4.5 \\
\hline & RDS & 1 & 1.5 \\
\hline & Invasive mechanical ventilation & 5 & 7.6 \\
\hline & Sepsis & 1 & 1.5 \\
\hline & Observation only & 11 & 16.7 \\
\hline Neonatal mortality & & 0 & 0 \\
\hline
\end{tabular}




\section{DISCUSSION}

In our research, 28/66 (42.4\%) women delivered vaginally and caesarean sections were performed in 38/66 (57.6\%) cases. Only $13.6 \%$ cases of cesarean section were undertaken for fetal distress as indication. 54 (81.8\%) women delivered at term, spontaneous early preterm birth occurred in 5 cases and in 7 cases elective cesarean section was performed before 37 weeks of gestation for obstetric reasons. There was a significant increase in caesarean deliveries $(52.76 \%)$ in the study by Pirjani et al in COVID19 infected laboring patients as compared to Non COVID19 ones. $^{6}$

In the study of eighteen articles by Zaigham et al $91 \%$ of the women were delivered by cesarean section. One neonatal death and one intrauterine death were also reported. Three maternal intensive care unit admissions were noted but no maternal deaths. ${ }^{7}$ In synopsis by Salem et al, SARS-CoV-2 infections in pregnancy have been associated with increased risk of admission to hospital but not with increased maternal and fetal death, and vertical utero-transmission. ${ }^{3}$

In this research, most of our cases were asymptomatic with only $13(19.7 \%)$ had mild respiratory or gastro intestinal symptoms on initial assessment at admission, including cough, sore throat, fever, weakness or diarrhea. COVID19 symptoms in pregnancy are similar to infections that occur in the general population in study by Huntley et al with respect to presenting symptoms of cough $(52 \%)$ and shortness of breath (30\%). Pregnant women, however, were less likely to report symptoms of headache $(41 \%)$, fever (34\%), chills (38\%), and diarrhoea (14\%) compared to the general population. ${ }^{8}$

In our study, only $16.7 \%$ COVID-19 positive patients presented with co morbidities. The cohort study by Villar et al suggested that COVID-19 positive pregnant females were at increased risk of maternal morbidities along with perinatal morbidity and mortality. ${ }^{9}$

The meta-analysis by Wei et al suggested strong association between COVID-19 positive pregnancy and pre-eclampsia, preterm births and fetal growth restriction. ${ }^{10}$

In our study, a total of $4(6.1 \%)$ newborn out of 66 deliveries came RT-PCR positive within 24 hours of delivery. Low birth weight seen in 15 cases, with extremely low birth weight seen in 2 in which Apgar score at $5 \mathrm{~min}$ is $<7$ is seen in one case. Out of 4 newborns, only one newborn developed symptoms (fever) and signs of dehydration within few hours of delivery, was transferred to NICU and recovered after three days of mechanical ventilation. The possibility of COVID-19 vertical transmission has been a significant concern in COVID-19 positive pregnant women, our study shows that there seems to be minimal risk $(6.1 \%)$ for vertical transmission to neonate. There were no cases of neonatal mortality.
The systematic review on 655 women by Walker et al reported that vertical transmission of COVID-19 is uncommon and is not greater if delivered vaginally or breastfed. $^{11}$

In study by Sindy et al, seven patients (36.8\%) were delivered via cesarean. 12 patients $(63.1 \%)$ presented in spontaneous labour, and $8(38.1 \%)$ had preterm delivery. No maternal intensive care unit admission, maternal sepsis, or maternal mortality was observed. Twenty-one neonates were evaluated for COVID-19 after birth. SARSCoV-2 RT-PCR test results were negative in $100 \%$ of the neonates. Thirteen neonates $(61.9 \%)$ were admitted to the neonatal intensive care unit. Prematurity was the most common cause of NICU admission 6 (46.1\%), with a length of stay of days. No invasive mechanical ventilation, neonatal sepsis, or neonatal mortality was observed. ${ }^{12}$

\section{CONCLUSION}

There was an increase in operative caesarean deliveries noted in our research in view of fear of vertical transmission in COVID-19 positive pregnant women and panic of monitoring high risk cases in Corona pandemic. There were no significant difference in COVID-19 related maternal mortality and morbidity as compared to nonpregnant women. Also, the vertical transmission rate was negligible.

\section{Limitation}

Our data was limited and cannot be generalized. More large-scale studies are required to reach a final conclusion. This study was limited mostly to women in their third trimester of pregnancy.

Funding: No funding sources Conflict of interest: None declared

Ethical approval: The study was approved by the Institutional Ethics Committee

\section{REFERENCES}

1. WHO. WHO coronavirus (COVID-19) dashboard, 2021. Available at: https://covid19.who. Accessed on 10 November 2021.

2. Royal College of Obstetricians and Gynaecologists. Coronavirus (COVID-19) infection in pregnancy, 2020. Available at: https://www.rcog.org.uk/globalassets/documents/gui delines/2020-07-24-coronavirus-covid-19infectioninpregnancy. Accessed on 10 November 2021.

3. Salem D, Katranji F, Bakdash T. COVID-19 infection in pregnant women: Review of maternal and fetal outcomes. Int J Gynaecol Obstet. 2021;152(3):291-8.

4. Dashraath P, Wong JLJ, Lim MXK, Lim LM, Li S, Biswas A, et al. Coronavirus disease 2019 (COVID19) pandemic and pregnancy. Am J Obstet Gynecol. 2020;222(6):521-31. 
5. Indian Council of Medical Research. Advisory on strategy for COVID-19 testing in India, 2020. Available at: https://www.icmr.gov.in/cteststrat.html. Accessed on 10 November 2021.

6. Pirjani R, Hosseini R, Soori T, Rabiei M, Hosseini L, Abiri A, et al. Maternal and neonatal outcomes in COVID-19 infected pregnancies: a prospective cohort study. J Travel Med. 2020;27(7):158.

7. Zaigham M, Andersson O. Maternal and perinatal outcomes with COVID-19: A systematic review of 108 pregnancies. Acta Obstet Gynecol Scand. 2020;99(7):823-9.

8. Huntley BJ, Huntley ES, Mascio D, Chen T, Berghella $\mathrm{V}$, Chauhan SP. Rates of maternal and perinatal mortality and vertical transmission in pregnancies complicated by severe acute respiratory syndrome coronavirus 2 (SARS-Co-V-2) infection: a systematic review. Obstet Gynecol. 2020;136(2):303-12.

9. Villar J, Ariff S, Gunier RB, Thiruvengadam R, Rauch S, Kholin A, et al. Maternal and Neonatal Morbidity and Mortality Among Pregnant Women With and Without COVID-19 Infection: The INTERCOVID Multinational Cohort Study. JAMA Pediatr. 2021;175(8):817-26.

10. Wei SQ, Bertrand M, Liu S, Auger N. The impact of COVID-19 on pregnancy outcomes: a systematic review and meta-analysis. CMAJ. 2021;193(16):5408.

11. Walker KF, Donoghue K, Grace N, Dorling J, Comeau JL, Li W, et al. Maternal transmission of SARS-COV-2 to the neonate, and possible routes for such transmission: a systematic review and critical analysis. BJOG. 2020;127(11):1324-36.

12. Moreno SC, To J, Chun H, Ngai IM. Vertical Transmission of COVID-19 to the Neonate. Infect Dis Obstet Gynecol. 2020;2020:8460672.

Cite this article as: Dholakiya S, Singh PS, Bamniya J, Doshi HU. Study of mode of delivery and maternal morbidity in preterm and term COVID-19 positive pregnant women. Int J Reprod Contracept Obstet Gynecol 2021;10:4383-7. 